\title{
ESTUDO DA CONSISTÊNCIA INTERNA E ANÁLISE FATORIAL EXPLORATÓRIA DA ESCALA CONTÍNUO DA SAÚDE MENTAL CCHS-MH EM AGRICULTORES DE PORTUGAL
}

\author{
Cláudia Balula Chaves \\ Health Shcool, CI\&DEI, Polytechnic Institute of Viseu, Portugal, \\ cchaves@essv.ipv.pt \\ Carlos Sequeira \\ Nursing School of Porto, CINTESIS, Porto - Portugal, \\ carlossequeira@esenf.pt \\ João Carvalho Duarte \\ Health School, UICISA: E, Polytechnic Institute of Viseu, Portugal \\ duarte.johnny@gmail.com \\ Paula Nelas \\ Health School, UICISA: E, Polytechnic Institute of Viseu, Portugal \\ pnelas@gmail.com \\ Emília de Carvalho Coutinho \\ Health School, UICISA: E, Polytechnic Institute of Viseu, Portugal \\ ecoutinho@essv.ipv.pt \\ Rui Manuel Dionísio \\ Agrupamento de Centors de Saúde Dão-Lafões -Unidade Local de Saúde Pública \\ rmtdsps@hotmail.com
}

Recepción Artículo: 27 agosto 2021 Admisión Evaluación: 07 septiembre 2021 Informe Evaluador 1: 12 septiembre 2021 Informe Evaluador 2: 13 septiembre 2021 Aprobación Publicación: 15 septiembre 2021

\section{RESUMO}

Os agricultores são um dos grupos ocupacionais com significativo risco de vulnerabilidade em termos de saúde mental, apresentando prevalência de morbidade psiquiátrica, em resultado dos efeitos dos fatores globais, nacionais, regionais, comunitários e locais. Estudar a consistência interna e análise fatorial exploratória da escala contínuo da saúde mental em agricultores e identificar que variáveis sociodemográficas dos agricultores que interferem na sua saúde mental. Estudo quantitativo, com corte transversal, descritivo analítico-correlacional. Os dados foram colhidos junto de 155 agricultores da região Norte e Centro de Portugal Continental, maioritariamente do género masculino (52,9\%), com uma média de idades de 53,41 anos ( $\pm 14,04$ anos). 0 instrumento de recolha de dados contém um questionário de caracterização sociodemográfica e a Escala Contínuo de Saúde Mental - CCHS-MH 2012. As variáveis sociodemográficas com relevância estatística são 0 género e a constituição do agregado familiar. As mulheres e os agricultores com agregado familiar de 3 elementos revelam melhor saúde mental. Após a realização da análise fatorial confirmatória para uma população de agricultores e depois da 


\section{ESTUDO DA CONSISTÊNCIA INTERNA E ANÁLISE FATORIAL EXPLORATÓRIA DA ESCALA CONTÍNUO DA SAÚDE MENTAL CCHS-MH EM AGRICULTORES DE PORTUGAL}

análise livre, caíram dois itens (4 e 5) porque $<0,44$, mas após a rotação varimax estes dois foram agrupados ao fator 3, os itens eliminados aproximam-se da resposta (tendência de resposta) do fator 3. Os participantes da contínuo da saúde mental apresentam índices mais elevados de bem-estar social e menos no bem-estar emocional, sugerindo-se uma política de literacia em saúde mental que abranja todo um conjunto de leis, programas, financiamentos, que estejam ao alcance de todos os agricultores.

Palavras chave: agricultores; saúde mental; fiabilidade

\section{ABSTRACT}

Internal consistency study and exploratory factor analysis of the continuum of mental health scale cchs-mh scale in farmers in Portugal. Farmers are one of the occupational groups at significant risk of vulnerability in terms of mental health, with a prevalence of psychiatric morbidity as a result of the effects of global, national, regional, community and local factors. Identify which sociodemographic variables of farmers that interfere with their mental health. Quantitative, cross-sectional, descriptive-analytical-correlational study. Data were collected from 155 farmers in the North and Central region of mainland Portugal, mostly male (52.9\%), with an average age of 53.41 years $( \pm 14.04$ years). The data collection instrument contains a sociodemographic questionnaire and the Continuous Mental Health Scale - CCHS-MH 2012. The sociodemographic variables with statistical significance are gender and the constitution of the household. Women and farmers with a 3-member household show better mental health. After performing the confirmatory factor analysis for a population of farmers and after the free analysis, two items ( 4 and 5 ) dropped because $<0.44$, but after varimax rotation these two were grouped to factor 3 , the eliminated items approximated if the response (response trend) of factor 3. Participants in the mental health continuum have higher rates of social well-being and less emotional well-being, suggesting a mental health literacy policy that covers a whole set of laws, programs, financing, which are available to all farmers.

Keywords: farmers; mental health; reliability

\section{INTRODUCCIÓN}

A Mental Health Continuum - Short Form (MHC-SF) é um instrumento de autorrelato para avaliação da saúde mental positiva, entendida como sintomas de afetos positivos, autodesenvolvimento e ligação social. Foi desenvolvida para colmatar a ausência de instrumentos que avaliassem as três dimensões da saúde mental positiva bem-estar emocional, psicológico e social (Keyes, 2002; Lamers, Westerhof, Bohlmeijer, Klooster \& Keyes, 2011). Foi elaborado como um instrumento para avaliar a saúde mental (Keyes, 2002) e continha em 40 itens distribuídos da seguinte forma: 7 correspondem à dimensão do bem-estar emocional, 18 para a dimensão do bem-estar psicológico e 15 para a dimensão do bem-estar social. A versão curta da escala (Continuum de Saúde Mental - Short Form, MHC-SF) contém 14 itens considerados protótipos das várias dimensões da saúde mental positiva. Keyes (2002) partiu do consenso de que a saúde mental não é apenas a ausência de doença mental, mas também inclui a presença de sentimentos positivos (bem-estar emocional) e funcionamento positivo na vida individual (bem-estar psicológico) e vida comunitária (bem-estar social).

Originalmente elaborado nos Estados Unidos (Keyes, 2002, 2005), o MHC-SF foi adaptado para países como a África do Sul (Keyes et al. 2008), Canadá (Peter, Roberts, \& Dengate 2011), Holanda (Westerhof \& Keyes, 2010), Itália (Petrillo, Capone, Caso, \& Keyes, 2014) e Irão (Joshanloo \& Nosratabadi, 2009).

Estudos que avaliaram o desempenho psicométrico do MHC-SF, utilizando dados exploratórios e análises fatoriais confirmatórias, mostram que uma estrutura com três fatores oblíquos é o mais adaptado para explicar 0 conjunto de itens que compõem o instrumento (Keyes, 2005; Keyes et al., 2008; Lamers, Westerhof, Bohlmeijer, ten Klooster, \& Keyes, 2011; Robitschek \& Keyes, 2009). Os mesmos estudos também verificaram que as subescalas do MHC-SF têm confiabilidade adequada. Apesar das correlações entre fatores serem moderadas a fortes. Os resultados indicaram que as três dimensões (bem-estar emocional, bem-estar psicológico e bem-estar social) podem ser distinguidas. Contudo, um estudo de Chen, Jing, Hayes e Lee (2012), que aplicou um modelo bidimensional, sugeriu que os indicadores de bem-estar emocional e bem-estar psicológico eram melhor explicados 
através de um fator geral do que por 2 fatores independentes. Por outras palavras, a variância comum compartilhada por todos os indicadores são maiores que a variância explicada por fatores específicos. No entanto, o modelo bidimensional ainda não foi aplicado a uma investigação conjunta das três dimensões da saúde mental positiva. Como tal, esta escala continua a ser tridimensional.

Este instrumento é composto por 14 itens que avaliam as dimensões supracitadas, constituindo assim uma escala tridimensional (Keyes et al., 2012; Keyes, et al., 2008; Lamers et al., 2011; Matos et al., 2010). Cada item representa 0 sentimento de bem-estar, bem como a frequência com que 0 experienciou, classificada numa escala tipo likert de 6 pontos, em que 6 representa "nunca" e 1 "todos os dias" (Joshanloo et al., 2013). Assim, na sua forma original, os itens encontram-se distribuídos pelas três dimensões, sendo o bem-estar emocional composto pelos itens 1, 2 e 3, o bem-estar social pelos itens 4, 5, 6, 7 e 8 e, finalmente, o bem-estar psicológico pelos itens $9,10,11,12,13$ e 14. No que se refere aos scores totais para cada dimensão, estes encontram-se entre os valores 3-18 no bem-estar emocional, 6-36 no bem-estar psicológico e, por fim, 5-30 no bem-estar social (Joshanloo et al., 2013). Este instrumento categoriza os indivíduos em três categorias - flourishing, languishing e saúde mental moderada. Para que estes sejam categorizados como flourishing devem pontuar com 5 ou 6 em pelo menos um dos três itens de bem-estar emocional e em, pelo menos, seis dos onze itens de funcionamento positivo - bem-estar social e bem-estar psicológico. Por seu turno, para que os indivíduos sejam classificados como languishing necessitam pontuar com 1 ou $2 \mathrm{em}$, pelo menos, um dos três itens de bem-estar emocional e em, pelo menos, seis dos onze itens de funcionamento positivo. Quando os indivíduos pontuam 3 ou 4 em pelo menos um dos três itens de bem-estar emocional e em, pelo menos, seis dos onze itens de funcionamento positivo são classificados com saúde mental moderada (Keyes, 2007). Inúmeros estudos em diferentes contextos culturais revelam a validade e confiança deste instrumento de avaliação, confirmando o modelo do duplo contínuo (Keyes et al., 2008; Lamers et al., 2011). Portanto, estes resultados corroboram a ideia de que a ausência de psicopatologia não implica necessariamente a presença de sentimentos positivos e um bom funcionamento individual e social, bem como 0 oposto (Lamers et al., 2011). Estudos realizados confirmam as três dimensões da saúde mental positiva proposta por Keyes em 2002, bem como a sua aplicabilidade em diferentes contextos culturais (Joshanloo et al., 2013; Keyes et al., 2008; Lamers et al., 2011). Para além disso, Lamers e colaboradores (2011) verificaram ainda que o MHC-SF é sensível a alterações e estável ao longo do tempo.

\section{OBJETIVOS DE LA INVESTIGACIÓN}

Estudar a consistência interna e análise fatorial exploratória da escala contínuo da saúde mental em agricultores e identificar que variáveis sociodemográficas dos agricultores que interferem na sua saúde mental.

\section{PARTICIPANTES}

Amostra de 155 agricultores, com uma idade mínima de 19 anos e máxima de 88 anos (média de 53,41 anos; $\pm 14,04$ ), em que 0 género masculino representa $52,9 \%$ da totalidade da amostra, maioritariamente com companheiro(a) (85,2\%), em que 0 agregado familiar é constituído por 4 ou mais elementos (46,5\%), havendo um claro predomínio de participantes que não têm filhos menores (64,5\%).

\section{METODOLOGíA}

Estudo quantitativo, com corte transversal, descritivo analítico-correlacional. 0 instrumento de recolha de dados contém um questionário de caracterização sociodemográfica e a Escala Contínuo de Saúde Mental CCHS-MH 2012. 0 estudo foi registado na Comissão Nacional de Proteção de Dados (Proc. nô 16612/2017). 0 protocolo de avaliação é de hétero-preenchimento, estando presentes elementos associados ao estudo para esclarecimentos de termos técnicos ou conceitos aos inquiridos. Cada protocolo de avaliação contemplava na folha de rosto, o consentimento informado com uma breve explicação do estudo, os fins a que se destinava e carácter voluntário do seu preenchimento e informados da confidencialidade servindo os dados apenas para fins estatísticos e para serem utilizados apenas pela equipa de investigação. Foi solicitado a assinatura aos inquiridos 
da folha de consentimento informado após terem anuído participar voluntariamente no estudo. 0 período de resposta ao protocolo de avaliação decorreu a partir do mês de outubro de 2017 até maio de 2018. Foram eliminados 12 questionários por não terem sido respondidos até ao seu terminus, ficando 155 completos.

\section{RESULTADOS}

A Tabela 1 apresenta as estatísticas e as correlações obtidas entre cada item e 0 valor global. Analisando os índices médios e respetivos desvios padrão dos diversos itens, verifica-se que se encontram na sua quase totalidade bem centrados, sendo o valor mínimo $(\mathrm{M}=1,95)$ foi obtido no item 2 "Interessado(a) com a vida", podendo este tornar-se 0 mais problemático quando relacionado com os restantes e o máximo no item $6(\mathrm{M}=3,62)$ "Que a nossa sociedade está a tornar-se num local melhor para todas as pessoas".

Em relação aos valores de alfa de Cronbach todos os itens podem classificar-se de muito bons, pois variam entre 0,913 e 0,919 . Os coeficientes de correlação item total corrigido revelam que 0 item $6(r=0,485)$ é 0 mais problemático não se revelando homogéneo face aos restantes itens. Calculado o valor de alfa de Cronbach, 0 mesmo revela-se muito bom, uma vez que se obteve um alfa global de 0,922.

Tabela 1 - Consistência interna dos itens da Escala Contínuo da Saúde Mental - CCHS-MH, da versão curta da MHC-SF (Keyes, 2002)

\begin{tabular}{l|l|l|l|l|l|l}
\hline $\mathbf{N}^{\mathbf{0}}$ Item & Itens & Média & $\mathbf{D p}$ & $\begin{array}{l}\mathbf{r} \text { item } \\
\text { total }\end{array}$ & $\mathbf{R}^{\mathbf{2}}$ & $\begin{array}{l}\boldsymbol{\alpha} \text { sem } \\
\text { item }\end{array}$ \\
\hline Durante o último mês, com que frequência sentiu-se... & & & & & \\
\hline $\mathbf{1}$ & Feliz & 2.30 & 1.107 & .673 & .660 & .916 \\
\hline $\mathbf{2}$ & Interessado(a) com a vida & 1.95 & 1.053 & .722 & .699 & .914 \\
\hline $\mathbf{3}$ & Satisfeito(a) com a vida & 2.12 & 1.093 & .768 & .762 & .913 \\
\hline $\mathbf{5}$ & $\begin{array}{l}\text { Que tinha algo de importante para contribuir } \\
\text { para a sociedade }\end{array}$ & 2.54 & 1.321 & .573 & .480 & .919 \\
\hline $\mathbf{6}$ & $\begin{array}{l}\text { Que pertencia à comunidade (como grupo social } \\
\text { ou ao bairro) }\end{array}$ & 2.29 & 1.339 & .668 & .571 & .916 \\
\hline $\mathbf{7}$ & $\begin{array}{l}\text { Que a nossa sociedade está a tornar-se num local } \\
\text { melhor para todas as pessoas }\end{array}$ & 3.62 & 1.522 & .485 & .682 & .923 \\
\hline $\mathbf{8}$ & $\begin{array}{l}\text { Que as pessoas ãa basicamente boas } \\
\text { lhe sentido que a nossa sociedade funciona faz- }\end{array}$ & 3.48 & 1.416 & .574 & .706 & .919 \\
\hline $\mathbf{9}$ & $\begin{array}{l}\text { Que gostava da maior parte dos aspetos da sua } \\
\text { personalidade }\end{array}$ & 2.26 & 1.185 & .704 & .670 & .914 \\
\hline $\mathbf{1 0}$ & $\begin{array}{l}\text { Que geria bem as responsabilidades da sua vida } \\
\text { quotidiana }\end{array}$ & 2.04 & 1.038 & .619 & .650 & .917 \\
\hline $\mathbf{1 1}$ & $\begin{array}{l}\text { Que tinha relacionamentos calorosos e de } \\
\text { confiança com os outros }\end{array}$ & 2.34 & 1.136 & .702 & .607 & .915 \\
\hline $\mathbf{1 2}$ & $\begin{array}{l}\text { Que tinha experiências que lhe desafiavam a } \\
\text { crescer e a tornar-se uma pessoa melhor }\end{array}$ & 2.45 & 1.285 & .689 & .589 & .915 \\
\hline $\mathbf{1 3}$ & $\begin{array}{l}\text { Confiante de forma a poder pensar ou exprimir } \\
\text { as suas próprias ideias e opiniões }\end{array}$ & 2.28 & 1.193 & .721 & .725 & .914 \\
\hline $\mathbf{1 4}$ & Que a vida tem sentido de direção e significado & 2.10 & 1.178 & .739 & .744 & .913 \\
\hline & \multicolumn{2}{|l}{} & & & $\mathbf{0 . 9 2 2}$ \\
\hline
\end{tabular}

Com a análise fatorial exploratória encontrou-se uma estrutura trifatorial que foi submetida à análise fatorial confirmatória. Como refere Marôco (2014), a análise fatorial confirmatória é uma técnica usada para avaliar a qualidade de ajustamento dum modelo de medida teórica à estrutura correlacional observada entre as variáveis manifestas (itens). Na tabela 2 são apresentadas as trajetórias dos itens com os respetivos fatores, bem como os rácios críticos e os coeficientes lambda. Assim, observa-se que todos os itens apresentam rácios críticos significativos, 
0 que leva à manutenção de todos eles. Por sua vez, os coeficientes lambda indicam que apenas os itens CSM4 e CSM5 correspondentes ao factor 2 apresentam saturações inferiores a 0.50, pelo que deve ser eliminado em futuras análises.

Tabela 2 - Rácios críticos e coeficientes lambda dos itens

\begin{tabular}{|c|c|c|c|c|c|c|}
\hline \multicolumn{2}{|c|}{ Trajectórias } & \multirow{2}{*}{$\begin{array}{l}\begin{array}{l}\text { Estimativ } \\
\text { as }\end{array} \\
.886\end{array}$} & \multirow{2}{*}{$\begin{array}{c}\text { Erro } \\
.092\end{array}$} & \multirow{2}{*}{$\begin{array}{l}\begin{array}{l}\text { Rácio } \\
\text { Critico }\end{array} \\
9.621\end{array}$} & \multirow{2}{*}{$\frac{\mathbf{p}}{* * *}$} & \multirow{2}{*}{$\begin{array}{l}\lambda \\
.753\end{array}$} \\
\hline $\begin{array}{l}\text { CSM } \\
10\end{array}$ & $\begin{array}{ll}<-- & \text { f1 } \\
- & \end{array}$ & & & & & \\
\hline $\begin{array}{l}\text { CSM } \\
11\end{array}$ & $\begin{array}{ll}<-- & \text { f1 } \\
- & \end{array}$ & .995 & .101 & 9.810 & $* * *$ & .773 \\
\hline $\begin{array}{l}\text { CSM } \\
12\end{array}$ & $\begin{array}{ll}<- & \text { f1 } \\
- & \text { f }\end{array}$ & 1.110 & .117 & 9.454 & $* * *$ & .762 \\
\hline $\begin{array}{l}\text { CSM } \\
13\end{array}$ & $\begin{array}{ll}<- & \text { f1 } \\
- & \end{array}$ & 1.169 & .107 & 10.888 & $* * *$ & .865 \\
\hline $\begin{array}{l}\text { CSM } \\
4\end{array}$ & $\begin{array}{ll}<- & F \\
- & 2\end{array}$ & 1.000 & & & & .393 \\
\hline $\begin{array}{l}\text { CSM } \\
5\end{array}$ & $\begin{array}{ll}<-- & F \\
- & 2\end{array}$ & .972 & .271 & 3.590 & $* * *$ & .377 \\
\hline $\begin{array}{l}\text { CSM } \\
6\end{array}$ & $\begin{array}{ll}<- & F \\
- & 2\end{array}$ & 2.439 & .507 & 4.814 & $* * *$ & .833 \\
\hline $\begin{array}{l}\text { CSM } \\
7\end{array}$ & $\begin{array}{ll}<- & F \\
- & 2\end{array}$ & 2.346 & .482 & 4.869 & $* * *$ & .861 \\
\hline $\begin{array}{l}\text { CSM } \\
8\end{array}$ & $\begin{array}{ll}<-- & F \\
- & 2\end{array}$ & 2.638 & .545 & 4.843 & $* * *$ & .921 \\
\hline $\begin{array}{l}\text { CSM } \\
1\end{array}$ & $\begin{array}{ll}<- & F \\
- & 3\end{array}$ & 1.000 & & & & .799 \\
\hline $\begin{array}{l}\text { CSM } \\
2\end{array}$ & $\begin{array}{ll}<-- & F \\
- & 3\end{array}$ & 1.039 & .085 & 12.293 & $* * *$ & .873 \\
\hline $\begin{array}{l}\text { CSM } \\
3\end{array}$ & $\begin{array}{ll}<-- & F \\
- & 3\end{array}$ & 1.120 & .088 & 12.725 & $* * *$ & .906 \\
\hline $\begin{array}{l}\text { CSM } \\
9\end{array}$ & $\begin{array}{ll}<-- & \text { f1 } \\
- & \end{array}$ & 1.000 & & & & .745 \\
\hline $\begin{array}{l}\text { CSM } \\
14\end{array}$ & $\begin{array}{ll}<-- & \text { f1 } \\
- & \text { f }\end{array}$ & 1.181 & .106 & 11.150 & $* * *$ & .885 \\
\hline
\end{tabular}

A figura 1 reporta-se ao modelo inicial onde se consta a distribuição dos itens por fator, os pesos fatoriais (saturações) dos itens e ainda a fiabilidade individual dos mesmos. Conforme referido anteriormente, os itens CSM4 e CSM5 revelam saturações inferiores aos valores de referência (0.50). Por sua vez, a fiabilidade individual, excluindo estes dois itens é superior a 0.25 , valor também tido como referência. Os índices de bondade de ajustamento global indicam que apenas a razão do qui quadrado com os graus de liberdade se apresenta adequado $(x 2 / g \mid=4.009)$. 


\section{ESTUDO DA CONSISTÊNCIA INTERNA E ANÁLISE FATORIAL EXPLORATÓRIA DA ESCALA \\ CONTÍNUO DA SAÚDE MENTAL CCHS-MH EM AGRICULTORES DE PORTUGAL}

Figura 1 - Modelo inicial com todos os itens distribuídos por fator

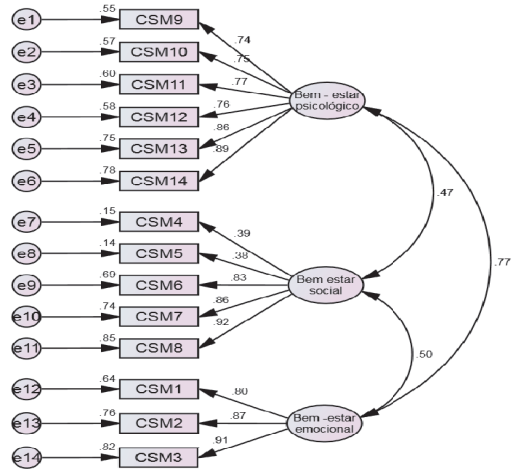

A figura 2 traduz o refinamento do modelo efectuado. Com a eliminação dos itens e com a correlação dos erros de medida dos itens CSM9 e CSM10 do fator 1 foi possível obter uma qualidade de ajustamento boa para $\left(x^{2} / \mathrm{gl}=2.127 ; \mathrm{GFI}=0.900 ; \mathrm{CFI}=0.959 ; \mathrm{RMR}=0.070 ; \mathrm{SRMR}=0.045\right)$. 0 (RMSEA=0.086) apresenta um valor centesimal ligeiramente acima do recomendado.

Figura 2 - Modelo final com índices de modificação e itens eliminados

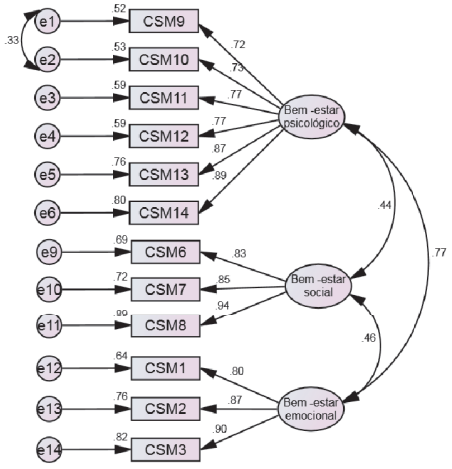

As correlações moderadas encontradas entre os fatores sugerem uma estrutura fatorial de $2^{a}$ ordem, a que se designou de Escala Contínuo da Saúde Mental - CCHS-MH, da versão curta da MHC-SF (Keyes, 2002) (CCHS-MH). Apurou-se que os itens do fator 1 explicam 74\% da variabilidade do CCHS-MM, sendo que o fator com maior percentagem de variância explicada é o fator 3 com $80 \%$ de variabilidade. Os índices de bondade de ajustamento global mantêm-se constantes em relação ao modelo final (cf. figura 3). 
Figura 3 - Modelo de $2^{\underline{a}}$ ordem

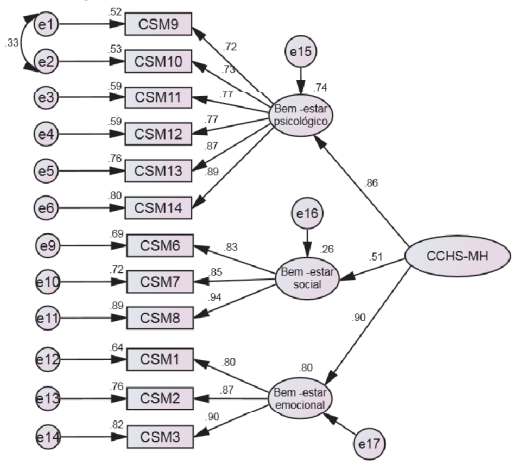

A tabela 3 expressa, em síntese, os índices de bondade de ajustamento global registados nos três modelos. É observável que no modelo inicial os índices de bondade de ajustamento são inadequados, enquanto no modelo com os itens eliminados e índices de modificação propostos pelo Amos e no modelo de $2^{a}$ ordem os mesmos índices se mostraram adequados.

Tabela 3 - Índices de bondade de ajustamento global dos diferentes modelos

\begin{tabular}{l|l|l|l|l|l|l}
\hline Modelo & $\boldsymbol{x}^{2 /}$ gl & GFI & CFI & $\begin{array}{l}\text { RMS } \\
\text { EA }\end{array}$ & RMR & $\begin{array}{l}\text { SRM } \\
\text { R }\end{array}$ \\
\hline Modelo inicial (figura 1) & 4.009 & 0.786 & 0.857 & 0.140 & 0.239 & 0.155 \\
\hline $\begin{array}{l}\text { Modelo com índices de } \\
\text { modificação figura 2 }\end{array}$ & 2.127 & 0.900 & 0.959 & 0.086 & 0.070 & 0.045 \\
\hline Modelo de segunda ordem & 2.127 & 0.900 & 0.959 & 0.086 & 0.070 & 0.045 \\
\hline
\end{tabular}

Termina-se o estudo da análise fatorial confirmatória fazendo referência à fiabilidade compósita (FC) e à variância extraída média (VEM). Em relação à primeira, os valores encontrados revelam uma fiabilidade compósita elevada dado que são superiores ao valor de referência (0.70). Quanto à VEM, um indicador de validade convergente dos fatores também são adequados uma vez que são superiores a 0.50 (valor de referência).

A fiabilidade compósita estratificada foi de 0.964 para uma variância extraída média de 0.692. A validade discriminante dos fatores foi avaliada pela comparação da VEM com os quadrados da correlação entre os fatores. Sendo os valores encontrados inferiores às VEM(s) respetivas, pode afirmar-se que os fatores têm validade discriminante (cf. tabela 4).

Tabela 4 - Fiabilidade compósita e variância extraída média

\begin{tabular}{l|l|l|l|l|l}
\hline Fatores & & & \multicolumn{3}{|c}{} \\
\cline { 4 - 6 } & F C & VEM & 1 vs2 & 1 vs3 & 2vs3 \\
\hline $\begin{array}{l}\text { Bem-estar } \\
\text { psicológico }\end{array}$ & 0.910 & 0.630 & 0.193 & 0.592 & \\
\hline $\begin{array}{l}\text { Bem- estar } \\
\text { social }\end{array}$ & 0.908 & 0.767 & & & 0.211 \\
\hline $\begin{array}{l}\text { Bem -estar } \\
\text { emocional }\end{array}$ & 0.895 & 0.740 & & & \\
\hline
\end{tabular}




\section{ESTUDO DA CONSISTÊNCIA INTERNA E ANÁLISE FATORIAL EXPLORATÓRIA DA ESCALA CONTÍNUO DA SAÚDE MENTAL CCHS-MH EM AGRICULTORES DE PORTUGAL}

Termina-se 0 estudo da análise psicométrica da escala com a exposição da consistência interna dos itens por fator, da validade convergente/divergente dos itens e matriz de correlação de Pearson.

No Bem-estar psicológico, verifica-se, pelos valores médios, homogeneidade nas respostas dadas aos

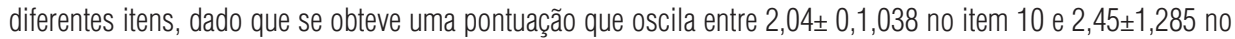
item 12. Os coeficientes de alpha de Cronbach dão indicações de boa e muito boa consistência interna, sendo 0 menor valor $(=0,886)$ encontrado no item 14 e o maior $(=0,904)$ no item 12 , com um alfa global de 0,912 , sugestivo de muito boa consistência interna.

Ao analisarem-se os resultados do Bem-estar social, constata-se que os índices médios oscilam entre os $3,48 \pm 1,416$ no item 7 e 3,62 $\pm 1,522$ no item 6 . Os coeficientes de alpha de Cronbach dão indicações de boa consistência interna, sendo o menor valor $(=0,827)$ o do item 8 e 0 maior $(=0,888)$ no item 6 , com um alfa total de 0,906, indicativo de muito boa consistência interna.

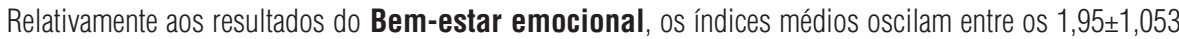
no item 2 e 2,30 $\pm 1,107$ no item 1. Os coeficientes de alpha de Cronbach dão indicações de boa consistência interna, sendo 0 menor valor $(=0,825)$ o do item 3 e 0 maior $(=0,882)$ no item 1 , com um alfa total de 0,894 , indicativo de boa consistência interna.

Tabela 5 - Consistência interna por subescalas

\begin{tabular}{|c|c|c|c|c|c|c|}
\hline $\begin{array}{l}\mathbf{N}^{0} \\
\text { Item }\end{array}$ & Itens & Média & $\mathrm{Dp}$ & $\begin{array}{l}\mathrm{r} / \text { item } \\
\text { total }\end{array}$ & $\mathrm{r} 2$ & $\begin{array}{l}\alpha \text { sem } \\
\text { item }\end{array}$ \\
\hline \multicolumn{7}{|c|}{ Fator 1 - Bem-estar psicológico } \\
\hline 9 & $\begin{array}{l}\text { Que gostava da maior parte dos aspetos } \\
\text { da sua personalidade }\end{array}$ & 2.26 & 1.185 & .710 & .561 & .902 \\
\hline 10 & $\begin{array}{l}\text { Que geria bem as responsabilidades da } \\
\text { sua vida quotidiana }\end{array}$ & 2.04 & 1.038 & .739 & .575 & .899 \\
\hline 11 & $\begin{array}{l}\text { Que tinha relacionamentos calorosos e } \\
\text { de confiança com os outros }\end{array}$ & 2.34 & 1.136 & .741 & .554 & .898 \\
\hline 12 & $\begin{array}{l}\text { Que tinha experiências que lhe } \\
\text { desafiavam a crescer e a tornar-se uma } \\
\text { pessoa melhor }\end{array}$ & 2.45 & 1.285 & .707 & .548 & .904 \\
\hline 13 & $\begin{array}{l}\text { Confiante de forma a poder pensar ou } \\
\text { exprimir as suas próprias ideias e } \\
\text { opiniões }\end{array}$ & 2.28 & 1.193 & .815 & .711 & .887 \\
\hline 14 & $\begin{array}{l}\text { Que a vida tem sentido de direção e } \\
\text { significado }\end{array}$ & 2.10 & 1.178 & .822 & .711 & .886 \\
\hline \multicolumn{2}{|c|}{ Coeficiente alfa Cronbach global } & \multicolumn{5}{|l|}{$\mathbf{0 , 9 1 2}$} \\
\hline \multicolumn{7}{|c|}{ Fator 2-Bem-estar social } \\
\hline 6 & $\begin{array}{l}\text { Que a nossa sociedade está a tornar-se } \\
\text { num local melhor para todas as pessoas }\end{array}$ & 3.62 & 1.522 & .788 & .634 & .888 \\
\hline 7 & Que as pessoas são basicamente boas & 3.48 & 1.416 & .797 & .655 & .880 \\
\hline 8 & $\begin{array}{l}\text { Que a forma que a nossa sociedade } \\
\text { funciona faz-lhe sentido }\end{array}$ & 3.59 & 1.489 & .857 & .736 & .827 \\
\hline \multicolumn{2}{|c|}{ Coeficiente alfa Cronbach global } & & & & \multicolumn{2}{|l|}{0,906} \\
\hline \multicolumn{7}{|c|}{ Fator 3-Bem-estar emocional } \\
\hline 1 & Feliz & 2.30 & 1.107 & .753 & .568 & .882 \\
\hline 2 & Interessado(a) com a vida & 1.95 & 1.053 & .804 & .660 & .839 \\
\hline 3 & Satisfeito(a) com a vida & 2.12 & 1.093 & .818 & .678 & .825 \\
\hline \multicolumn{2}{|c|}{ Coeficiente alfa Cronbach global } & \multicolumn{5}{|l|}{0,894} \\
\hline
\end{tabular}

A matriz de correlação entre os quatro fatores e o global da escala indica que as correlações são positivas e significativas. Perante estes resultados, apura-se que o aumento ou diminuição dos índices da Escala Contínuo 
da Saúde Mental num dos fatores se encontra associado a aumento ou diminuição nos índices da saúde mental nos restantes fatores (cf. Tabela 6).

Tabela 6 - Matriz de Correlação de Pearson entre os fatores da Escala Contínuo da Saúde Mental - CCHS-MH, da versão curta da MHC-SF (Keyes, 2002)

\begin{tabular}{lllll}
\hline Fatores & $\begin{array}{l}\text { Bem-estar } \\
\text { psicológico }\end{array}$ & $\begin{array}{l}\text { Bem-estar } \\
\text { social }\end{array}$ & $\begin{array}{l}\text { Bem-estar } \\
\text { emocional }\end{array}$ & $\begin{array}{l}\text { Fator } \\
\text { global }\end{array}$ \\
\hline $\begin{array}{l}\text { Bem-estar } \\
\text { psicológico }\end{array}$ & - & $.394^{* *}$ & $.701^{* *}$ & $.899^{* *}$ \\
$\begin{array}{l}\text { Bem-estar social } \\
\begin{array}{l}\text { Bem-estar } \\
\text { emocional }\end{array}\end{array}$ & $.394 * *$ & - & $.412^{* *}$ & $.716^{* *}$ \\
Fator global & $.701^{* *}$ & $.412^{* *}$ & - & $.824 * *$ \\
\hline
\end{tabular}

$* \mathrm{p}>0,05 * * \mathrm{p}>0,001$

\section{DISCUSIÓN}

Considera-se, como limitação metodológica o facto de os participantes terem sido recrutados de acordo com o processo de amostragem em bola de neve que não permitiu determinar o erro de amostragem ou fazer inferências sobre a população da amostra obtida, o que pode ter acarretado vieses dos resultados. Não obstante, julgase que 0 tamanho da amostra é satisfatorio traduzindo-se em resultados fiáveis, embora, não sendo representativa, condiciona a generalização dos resultados a outra população de agricultores. Como tal, o presente estudo carece de confirmação com a realização de outros estudos na mesma área, caso possível, com amostras mais dilatadas e que sejam representativas da população em estudo.

A questão de investigação previa verificar que variáveis sociodemográficas interferem na saúde mental dos agricultores, tendo-se constatado que o género e a constituição do agregado familiar foram as variáveis que revelaram relevância estatística. Assim, concluiu-se que as mulheres revelam melhor saúde mental, com destaque para 0 bem-estar social $(M=55,15 ; \pm 26,13)$, resultando em diferenças estatisticamente significativas no bem-estar psicológico ( $p=0,003)$, bem-estar emocional $(p=0,000)$ e na saúde mental global $(p=0,001)$. Os agricultores cujo agregado familiar é constituído por 3 elementos são os que manifestam melhor saúde mental, enquanto os agricultores com 4 ou mais elementos na constituição do seu agregado familiar revelam menor saúde mental, com diferenças estatisticamente significativas no bem-estar psicológico ( $p=0,025)$, bem-estar social $(p=0,000)$ e na saúde mental global ( $\mathrm{p}=0,012)$. No estudo de Liu et al. (2017), com uma amostra de 3675 agricultores, 0 género, a idade, 0 estado civil, o nível de escolaridade e o rendimento familiar mensal influenciaram a qualidade de vida relacionada com a saúde dos agricultores. Os autores concluíram que os agricultores do género masculino foram os que demonstraram pior qualidade de vida relacionada com a saúde, resultados corroborados pelo presente estudo, uma vez que se constatou que são as mulheres agricultoras que revelam melhor saúde mental, o que sugere melhor qualidade de vida relacionada com a saúde.

\section{CONCLUSIONES}

0 perfil do agricultor neste estudo revela uma idade média de 53,41 anos ( $\pm 14,04$ anos), sobressaindo os participantes com idade compreendida entre os 41-65 anos, estando em maioria os homens, os participantes com companheiro/a, que possuem um agregado familiar constituído por 4 ou mais elementos, com um claro predomínio de agregados familiares sem menores. As variáveis sociodemográficas que interferem na saúde mental dos agricultores são 0 género e a constituição do agregado familiar. A matriz de correlação entre os quatro fatores e 0 global da escala indica que as correlações são positivas e significativas. Perante estes resultados, apura-se que 0 aumento ou diminuição dos índices da Escala Contínuo da Saúde Mental num dos fatores se encontra associado a aumento ou diminuição nos índices da saúde mental nos restantes fatores. 


\section{ESTUDO DA CONSISTÊNCIA INTERNA E ANÁLISE FATORIAL EXPLORATÓRIA DA ESCALA CONTÍNUO DA SAÚDE MENTAL CCHS-MH EM AGRICULTORES DE PORTUGAL}

Após a realização deste estudo, sugere-se que os enfermeiros de saúde comunitária desenvolvam ações de formação sobre a saúde mental na comunidade agrícola, como meio de dotar os agricultores, independentemente da dimensão da sua exploração, de literacia em saúde mental, para que estes fiquem mais despertos para todos os fatores de risco e formas de procurar ajuda. É que a pessoa com menos literacia terá menos oportunidades de procurar ajuda em termos de saúde. Será importante que essas ações de formação sejam adequadas às características sociodemográficas e contextuais da população alvo. É que a saúde é um valor partilhado por todos os cidadãos quer nos países desenvolvidos, quer nos países em desenvolvimento; é a mais importante condição para a vida humana mais do que o sucesso económico. A saúde é também um fenómeno com forte componente social, psicológico, espiritual e físico, pelo que não existe saúde, sem saúde mental. Consequentemente, a saúde mental é uma condição prévia para a produtividade laboral e para a competitividade das sociedades.

Sugere-se também a criação de grupos de autoajuda, fornecendo sistemas de apoio e de acesso fácil aos agricultores, nos centros de saúde, com equidade social e justa; dotar os agricultores de conhecimentos para que possam desenvolver ambientes saudáveis de trabalho; promover apoio psicológico aos agricultores sempre que necessário; valorizar-se mais o seu trabalho e conceder-Ihes reforços positivos, proporcionando o equilíbrio laboral, pessoal e familiar e, consequentemente, melhor saúde mental.

\section{REFERENCIAS BIBLIOGRÁFICAS}

Keyes, C. L. M. (2007). Promoting and protecting mental health as flourishing: a complementary strategy for improving national mental health. The American Psychologist, 62, 95-108.

Liu, X., Gu, S., Duan, S., Wu, Y., Y., C., Wang, J., \& Dong, H. (2017). Comparative Study on Health-Related Quality of Life of Farmers and Workers. Value in Health Regional, 12, 123-129. https://doi.org/10.1016/j.vhri.2017.03.006 